\title{
A CONTRIBUTION TO THE IDENTIFICATION OF THE PSEUDONYMS USED IN THE MINUTES AND REPORTS OF THE COMMUNIST INTERNATIONAL*
}

The historian who studies the history of the Comintern has to rely mainly on printed sources. For this purpose the published minutes and reports of the seven Congresses of the Comintern (1919-35) and the thirteen plenary sessions of its Executive Committee (1922-33) are of particular interest.

The use of these minutes and reports is rendered difficult by the fact that a number of the participants did not act under their real name, respectively under the assumed one they made use of permanently, but under pseudonyms. The pseudonyms that were used over a long period cause hardly any problem (e.g., the pseudonym Karski, which was used by Julian Marchlewski already from the 1890's). These pseudonyms are generally known. Little is known, however, about those chosen ad hoc. Up to now no systematic attempt has been made to decipher them.

The results of my research, summarized in the following list, show that such an attempt certainly is not hopeless. The list contains all the pseudonyms occurring in the minutes and reports that I have been able to identify. I have confined my study to the pseudonyms of persons who spoke or were elected on a commission; so I have not taken into account the pseudonyms that occur only on the lists of participants. In general, I have made use of the German version; for the 9th ECCI plenum the report in English was consulted, for the 11th, 12th and 13th plenary sessions the Russian version of the minutes was used. When different pseudonyms occur in other versions that were at my disposal, they are mentioned in the footnotes, as far as I was able to decipher them.

The list contains the pseudonyms of nearly all the leading officials of the international Communist movement, but also those of most officials who were less important. It shows that it happened 1) that different persons used the same pseudonym at different Congresses or plenary sessions, 2)

* This contribution is a sequel to my article "The Communist International, 1919-43: The Personnel of its Highest Bodies", in: International Review of Social History, XXI (1976), pp. 151-85. 
that different persons used the same pseudonym at the same Congress or plenary session, 3) that the same person used different pseudonyms at different Congresses or plenary sessions, and 4) that the same person used different pseudonyms at the same Congress or plenary session.

To give some examples: the speudonym Seemann was used by the delegate of the Mexican CP Charles Philips at the Second Congress in 1920 and by the delegate of the KAPD (Communist Workers' Party of Germany) Bernhard Reichenbach at the Third Congress in 1921; at the Sixth Congress in 1928 Charles Philips represented the CP of the USA under the pseudonym Gómez. The delegate of the CP of Germany at the Fifth Congress in 1924 Josef Winternitz gave his address under the name Ternik, while he acted under the pseudonym Sommer in the Committees. At the same Congress Fedor Raskol'nikov represented the CP of the Soviet Union under his own name and the $\mathrm{CP}$ of China under the name Petrov in the Commission for national and colonial affairs; further he represented the $\mathrm{CP}$ of the Soviet Union under his own name in the Commission for British affairs and under the name Petrov in the one for Japanese affairs. David Lipec represented the $\mathrm{CP}$ of Great Britain under the name Bennett and the $\mathrm{CP}$ of the Soviet Union under the pseudonym Petrovskij at the Fifth Congress. The latter pseudonym was used at the same Congress by the delegate of the CP of Lithuania Karl Pojel.

From these examples it is clear that one person often represented different parties under different pseudonyms. Thus the decipherment of the pseudonyms does not only lead to the identification of several speakers or commission members, but also gives an insight into the close personal relations between the delegations of the different parties. In view of the influence of these bodies on the policies of national parties it is hardly necessary to point out how important it is for the study of the history of the national Communist parties to make sure who said what at the Congresses of the Comintern or the plenary sessions of the ECCI.

As I stated in my article "The Communist International, 1919-43", the use of pseudonyms did not only serve the purpose of protecting the delegates against persecution, but increasingly that of manipulating the organs of the Comintern and its sections, especially during Stalin's struggle against the Trockij-Zinov'ev opposition and against Bucharin. For example, the secretary-general of the CP of Rumania Baltazar, whose real name was Elek Köblös, was recalled in 1928 because of right-wing deviation; his successor was a man called Barbu, who acted under the pseudonym Petrulescu at the Sixth Congress. Barbu-Petrulescu's real name was Vitalij Holostenko; from 1922 till 1928 he had belonged to the CP of the Ukraine; in 1931 he was recalled because of left-wing deviation. He was succeeded 
by a certain Horn, whose real name was Aleksander Danieluk and who came originally from Warsaw. Until 1929 he had been one of the leading officials of the CP of Poland; at the Fifth Congress he had represented this party under the pseudonym Stefanski. As a result of his belonging to the Warski-Kostrzewa group he was deprived of all his functions in the CPP and transferred to the Comintern apparatus. At the 13th ECCI plenum he represented the $\mathrm{CP}$ of Rumania under the pseudonym Grigorescu.

The practice illustrated by this example often was contrary to the rules of the Comintern and its sections. For instance, it was not permitted that members of the International Control Commission were elected on other organs of the Comintern; to evade this rule the delegate of the CP of Italy Egidio Gennari, who had belonged to the ICC from the Fifth Congress, was elected to the Presidium and the Political Secretariat of the ECCI at the 8th plenum under the pseudonym Maggi and not under his own name.

It goes without saying that it would have been desirable for me to have shown the way in which I arrived at the identification of each pseudonym, but this is impossible for reasons of space. In the first place I base my study on the available publications of the Comintern itself, which are not always consistent in the use of the pseudonyms, secondly, on the information of people who were themselves active in the organs of the Comintern or played an active part in the Communist movement of their country and, finally, on the information of authors who had or still have access to the relevant archives.

As a result of a systematic study of the publications mentioned, by comparing and combining the information they give, helped by personal knowledge, I have been able to identify most, but not all the pseudonyms occurring in the minutes and reports. The following list has by no means the pretention to be complete. Especially in the case of the representatives of the small parties in Asia, Africa and Latin America it was not always clear whether the name under which they acted was their real name. I need not emphasize either that my identification is not necessarily the last word. Nonetheless, I hope that the list below will be a useful contribution to research into the history of the Comintern.

The pseudonyms have been arranged in alphabetical order for each Congress and plenary session. The real names are given in the second column, while in the third column the section is mentioned that the person in question represented at the time. The pseudonyms are partly written in a different spelling from the one in the minutes - either as usual in the country of origin (e.g., Queiros instead of Keyros, Próżański instead of Prusanski) or in the accepted transcription (e.g., Bosković instead of Boschkowitz or Boschkowitsch, Hadjar instead of Hadschar); when my 
spelling differs considerably from the one in the minutes, so that this might lead to doubt, the latter has been inserted into the list.

First Congress (2-6 March 1919) ${ }^{1}$

$\begin{array}{lll}\text { Albert, Max } & \text { Eberlein, Hugo } & \text { Germany } \\ \text { Gruber } & \text { Steinhardt, Karl } & \text { Austria }\end{array}$

Second Congress (19 July - 7 August 1920) ${ }^{2}$

$\begin{array}{lll}\text { Allan, Helen } & \text { Roy, Evelyn } & \text { Mexico } \\ \text { Allan, Robert } & \text { Roy, Manabendra Nath } & \text { Mexico } \\ \text { Artem } & \text { Sergeev, Fedor A. } & \text { Russia } \\ \text { Berzin } & \text { Berzin-Anderson, Jan } & \text { Latvia } \\ \text { Blank, Paul } & \text { Blank-Berzin, Paul } & \text { Latvia } \\ \text { Fraina } & \text { Corey, Lewis } & \text { USA } \\ \text { Hassanov } & \text { Farsi, Kerim-Aga } & \text { Persia } \\ \text { Lao } & \text { Lao.Hsiu-chao } & \text { China } \\ \text { Maring } & \text { Sneevliet, Hendricus J. F. M. } & \text { Indonesia } \\ \text { Men'šoj } & \text { Levin, Lev S. } & \text { Russia } \\ \text { N } & \text { Conolly, Roddy } & \text { Ireland } \\ \text { Pak } & \text { Pak Chin-sun } & \text { Korea } \\ \text { Sablin, Nikolaj } & \text { Nedjalkov, Ivan } & \text { Bulgaria } \\ \text { Seemann } & \text { Philips, Charles } & \text { Mexico } \\ \text { Sultan-Zade, A. } & \text { Mikaeljan, Avetis } & \text { Persia } \\ \text { Vanini, Lorenzo } & \text { Minev, Stojan } & \text { France } \\ \text { Viktor } & \text { Krastýn, Karl } & \text { Latvia } \\ \text { X } & \text { McAlpine, Eamon } & \text { Ireland }\end{array}$

${ }^{1}$ Der I. Kongress der Kommunistischen Internationale. Protokoll der Verhandlungen in Moskau vom 2. bis zum 19. März 1919 (Petrograd, 1920).

2 Der zweite Kongress der Kommunist. Internationale. Protokoll der Verhandlungen vom 19. Juli in Petrograd und vom 23. Juli bis 7. August 1920 in Moskau (Hamburg, 1921); Berichte zum Zweiten Kongress der Kommunist. Internationale (Hamburg, 1921). ${ }^{3}$ M. N. Roy from India, who lived in Mexico in the years 1917-19, had founded the CP of Mexico in 1919 at the direction of Borodin (Michail Grusenberg), who acted as Comintern emissary in Mexico at the time.

4 Stojan Minev, alias Lorenzo Vanini, alias Lebedev, alias Stepanov, alias Carlo Magno, alias George Chavaroche, alias Moreno, alias Iren, was born in Bulgaria in 1891. Until the dissolution of the Comintern in 1943 he was active in its organs. In the years 1931-33 and 1937-39 he was a Comintern emissary in Spain. During World War II he worked as ECCI representative in Latin America. He did not return home after the war. In the years 1945-58 he was at the Institute of Economics of the Academy of Sciences of the USSR, and after 1958 at the Institute of Social Sciences of the Gentral Committee of the CP of the Soviet Union. He died in Moscow in May 1959. 
Third Congress (22 June - 12 July 1921) ${ }^{5}$

$\begin{array}{lll}\text { Agasada } & \text { Agha-Zade } & \text { Persia } \\ \text { Ballister } & \text { Minor, Robert } & \text { USA } \\ \text { Bergmann } & \text { Meyer } & \text { Germany } \\ \text { Brand } & \text { Lauer, Henryk } & \text { Poland } \\ \text { Friesland } & \text { Reuter, Ernst } & \text { Germany } \\ \text { Gliński } & \text { Królikowski, Stefan } & \text { Poland } \\ \text { Hempel } & \text { Appel, Jan } & \text { Germany } \\ \text { Kasjan } & \text { Ter-Kasparjan, Sarkis } & \text { Armenia } \\ \text { Marshall } & \text { Bedacht, Max } & \text { USA } \\ \text { Michaljak } & \text { Warszawski, Adolf } & \text { Poland } \\ \text { Rwal } & \text { Reicher, Gustaw } & \text { Poland } \\ \text { Sachs } & \text { Schwab, Alexander } & \text { Germany } \\ \text { Seemann } & \text { Reichenbach, Bernhard } & \text { Germany } \\ \text { Takeguschi } & \text { Tagushi Unzo } & \text { Japan } \\ \text { Walecki } & \text { Horwitz, Maksymilian } & \text { Poland } \\ \text { Zadek, Djavad } & \text { Djavadzade } & \text { Persia }\end{array}$

Fourth Congress (5 November - 5 December 1922)

$\begin{array}{lll}\text { Billings } & \text { Huiswoud, Otto } & \text { USA } \\ \text { Carr } & \text { Katterfeld, Ludwig } & \text { USA } \\ \text { Domski } & \text { Stein, Henryk } & \text { Poland } \\ \text { Jansen } & \text { Proost, Johannes } & \text { Holland } \\ \text { Keller } & \text { Fiedler, Franciszek } & \text { Poland } \\ \text { Kostrzewa } & \text { Koszutska, Maria } & \text { Poland } \\ \text { Lansing } & \text { Foster, William Z. } & \text { USA } \\ \text { Marshall } & \text { Bedacht, Max } & \text { USA } \\ \text { Michalkowski } & \text { Warszawski, Adolf } & \text { Poland } \\ \text { Radić } & \text { Radovanović, Ljubomir } & \text { Yugoslavia } \\ \text { Saša } & \text { Stokes, Rose Pastor } & \text { USA } \\ \text { Stanić } & \text { Novaković, Kosta } & \text { Yugoslavia } \\ \text { Walecki } & \text { Horwitz, Maksymilian } & \text { Poland } \\ \text { Warski } & \text { Warszawski, Adolf } & \text { Poland }\end{array}$

5 Protokoll des III. Kongresses der Kommunistischen Internationale (Moskau, 22. Juni bis 12. Juli 1921) (Hamburg, 1921).

6 Protokoll des Vierten Kongresses der Kommunistischen Internationale, PetrogradMoskau vom 5. November bis 5. Dezember 1922 (Hamburg, 1923); Inprekorr (German edition), Vol. 2 (1922), Nos 223-24, 226, 229-32, 236-37, 240; the representative of the CP of the USA Saša is mistakenly named as representative of the CPGB ibid., No 236, p. 1755. 
Fifth Congress (17 June -8 July 1924)

\begin{tabular}{|c|c|c|}
\hline Alonso & Felisien, Alonso & Spain \\
\hline Badulescu & Moskovici, Gelbert & Rumania \\
\hline Bär & Winterich, Jean & Germany \\
\hline Bennett & Lipec, David & Britain \\
\hline Bini & Bibolotti, Aladino & Italy \\
\hline Bošković & Filipović, Filip & Yugoslavia \\
\hline Brand & Lauer, Henryk & Poland \\
\hline \multicolumn{3}{|l|}{ Brandt, see Brand } \\
\hline Buţureanu & Fabian, David & Rumania \\
\hline \multicolumn{3}{|c|}{ Buzureanu, see Buţureanu } \\
\hline Christophe & Humbert-Droz, Jules & Switzerland \\
\hline \multicolumn{3}{|c|}{ Contieras, see Contreras } \\
\hline Contreras & Vidali, Vittorio & Argentina \\
\hline Dunajewski & Amsterdam, Saul & Poland \\
\hline Edwards & Carlson, Oliver (?) & USA \\
\hline Ercoli & Togliatti, Palmiro & Italy \\
\hline Ferri & Leonetti, Alfonso & Italy \\
\hline Freimuth & Remmele, Hermann & Germany \\
\hline Gebhardt & Geschke, Ottomar & Germany \\
\hline Georgescu & Crisan, Aleksander & Rumania \\
\hline \multicolumn{3}{|l|}{ Giraud, see Girault } \\
\hline Girault, Suzanne & Depollier, Suzanne & France \\
\hline Górski & Graezer, Konstanty & Poland \\
\hline Grzegorzewski & Grzelczak, Franciszek & Poland \\
\hline Hanada & Sano Manabu & Japan \\
\hline Heinz & Neumann, Heinz & Germany \\
\hline Herzog & Heckert, Fritz & Germany \\
\hline Kalina & Leszczyński, Julian & Poland \\
\hline Kleine & Guralski, Abraham & Germany \\
\hline Krajewski & Stein, Wladyslaw & Poland \\
\hline Kulen & Culen, Marek & Czechoslovakia \\
\hline Lazić & Stefanović, Lazar & Yugoslavia \\
\hline Leński & Leszczyński, Julian & Poland \\
\hline Linde & Schlecht, Paul & Germany \\
\hline Lothar & Jakobs, Hermann & Germany \\
\hline
\end{tabular}

7 Protokoll Fünfter Kongress der Kommunistischen Internationale (Hamburg, n.d.); Fifth Congress of the Communist International. Abridged Report of Meetings held at Moscow June 17th to July 8th, 1924 (London, n.d.). In the English version the pseudonym Brown is used for James Larkin (Ireland) and the pseudonym Schulze for Wilhelm Schumacher (Germany). The pseudonym Braun is used for Ernst Thälmann (Germany). 


$\begin{array}{lll}\text { Marinović } & \text { Kaclerović, Triša } & \text { Yugoslavia } \\ \text { Ordon } & \text { Dąbal, Tomasz } & \text { Poland } \\ \text { Pepper } & \text { Pogány, József } & \text { USA } \\ \text { Petrov } & \text { Raskol'nikov, Fedor F. } & \text { USSR } \\ \text { Petrovskij } & \text { Lipec, David } & \text { USSR } \\ \text { Petrovskij } & \text { Pojel, Karl } & \text { Lithuania } \\ \text { Philipps } & \text { Aronberg, Philip } & \text { USA } \\ \text { Piccini } & \text { Mersú, Gustavo } & \text { Italy } \\ \text { Popescu } & \text { Stefanov, Boris } & \text { Rumania } \\ \text { Rienzi } & \text { Tasca, Angelo } & \text { Italy } \\ \text { Rossi } & \text { Grieco, Ruggero } & \text { Italy } \\ \text { Salcki } & \text { Horwitz, Maksymilian } & \text { Poland } \\ \text { Senko } & \text { Copić, Vladimir } & \text { Yugoslavia } \\ \text { Skulski } & \text { Mertens, Stanislaw } & \text { Poland } \\ \text { Skutzki, see Skulski } & & \\ \text { Sommer } & \text { Winternitz, Josef } & \text { Germany } \\ \text { Stanislawski } & \text { Kwiatkowski, Waclaw (?) } & \text { Poland } \\ \text { Stefański } & \text { Danieluk, Aleksander } & \text { Poland } \\ \text { Stepansk, see Stefański } & & \\ \text { Stirner } & \text { Woog, Edward } & \text { Mexico } \\ \text { Tani } & \text { Kondo Eizo } & \text { Japan } \\ \text { Ternik } & \text { Winternitz, Josef } & \text { Germany } \\ \text { Ulmer } & \text { Dengel, Philipp } & \text { Germany } \\ \text { Vasilkiv } & \text { Krilyk, Osip } & \text { Poland } \\ \text { Walecki } & \text { Horwitz, Maksymilian } & \text { Poland } \\ \text { Warski } & \text { Warszawski, Adolf } & \text { Poland } \\ \text { Wassilkow, see Vasilkiv } & \\ \text { Willi } & \text { Münzenberg, Willi } & \text { Germany }\end{array}$

Sixth Congress (17 July -1 September 1928) ${ }^{9}$

$\begin{array}{lll}\text { Agić } & \text { Brezović, Matija } & \text { Yugoslavia } \\ \text { Alphonso } & \text { Tohir, Mohamed } & \text { Indonesia } \\ \text { An } & \text { Nguyen-Van-Tao } & \text { Indochina }\end{array}$

${ }^{8}$ In the Russian version Gordon is given instead of Ordon. Pjatyj Vsemirnyj Kongress Kommunističeskogo Internacionala, 17 ijunja -8 ijulja 1924 g., Stenografičeskij otčet (Moscow, Leningrad, 1925), I, pp. 764-66; II, pp. 241-42.

9 Protokoll Sechster Weltkongress der Kommunistischen Internationale, Moskau, 17. Juli - 1. September 1928 (Hamburg, Berlin, 1928); VI Kongress Kominterna, Stenograficeskij otcet (Moscow, Leningrad, 1929). In the Russian version the pseudonym Stancu is used for Ion Heigl (Rumania) and the pseudonym Baltazar for Elek Köblös (Rumania). 
Balthario

Banderas

Bennett

Bielewski

Brand

Chang Piao

Chen Kwang

Choraz

Contreras

Ercoli

Gallo

Garlandi

Gómez

Grünbaum

Hadjar

Hanin

Hosoi

Jones

Kato

Kemény

Korczik

Kostrzewa

Li-Kuang

Lidin

Limanowski

Lovickij

Mahmud

Manavar

Mehring

Mikolos

Najdenov

Nakovanović

Narayan

Omura

Padi-Animin

Pepper

Petrulescu

Próżański

Raza

Rienzi

Rogić
Köblös, Elek

Pestkowski, Stanislaw

Lipec, David

Paszyn, Jan

Lauer, Henryk

Chang Kuo-tau

Chou En-lai

Volek, Július

Vidali, Vittorio

Togliatti, Palmiro

Longo, Luigi

Grieco, Ruggero

Philips, Charles

Szapiro, Nathan

Awerbach, Wolf

Heigl, Ion

Ichikawa Shoichi

Hall, Otto

Sano Manabu

Révai, József

Lohinowicz, Józef

Koszutska, Maria

Su Chao-cheng

Hlebec, Albert

Matys, Roman

Popov, Nikolaj N.

Ahmed, Habib (?)

Musso

Mirring, Richard

Skrypnik, Nikolaj A.

Volek, Július

Kovačević, Nikola

Tagore, Saumendra Nath

Takahashi Sadaki

Alimin Mas P.

Pogány, József

Holostenko, Vitalij

Olszewski, Aleksander

Shafik, Mohamed

Tasca, Angelo

Žorga, Jakob
Rumania

Poland

Britain

Poland

Poland

China

China

Czechoslovakia

Mexico

Italy

Italy

Italy

USA

Poland

Palestine

Rumania

Japan

USA

Japan

Hungary

Poland

Poland

China

Yugoslavia

Poland

UkrSSR

India

Indonesia

Estonia

UkrSSR

Czechoslovakia

Yugoslavia

India

Japan

Indonesia

USA

Rumania

Poland

India

Italy

Yugoslavia 


$\begin{array}{lll}\text { Rossi } & \text { Grieco, Ruggero } & \text { Italy } \\ \text { Samin } & \text { Darsono } & \text { Indonesia } \\ \text { Saris } & \text { Siantos, Giorgio (?) } & \text { Greece } \\ \text { Sikandar } & \text { Usmani, Shaukat } & \text { India } \\ \text { Spachić } & \text { Vuković, Gojko } & \text { Yugoslavia } \\ \text { Strachov } & \text { Chü Chiu-pai } & \text { China } \\ \text { Sultan-Zade } & \text { Mikaeljan, Avetis } & \text { Persia } \\ \text { Travin } & \text { Gusev, Sergej } & \text { USSR } \\ \text { Tsiu Vito } & \text { Chü Chiu-pai } & \text { China } \\ \text { Vries, } \text { A. de } & \text { Leeuw, Alex de } & \text { Holland } \\ \text { Williams } & \text { Michailov, Boris } & \text { USSR } \\ \text { Witkowski } & \text { Landy, Adam } & \text { Poland }\end{array}$

Seventh Congress (25 July - 20 August 1935) ${ }^{10}$

$\begin{array}{lll}\begin{array}{l}\text { Albino } \\ \text { André }\end{array} & \text { Gonçalves, Bento Antonio } & \text { Portugal } \\ \text { Baldur } & \text { Cvijić, Stjepan } & \text { Yugoslavia } \\ \text { Battista } & \text { Rudolf, Fritz } & \text { Germany } \\ \text { Bielewski } & \text { Ciufoli, Domenico } & \text { Italy } \\ \text { Billet } & \text { Paszyn, Jan } & \text { Poland } \\ \text { Borches } & \text { Docker, Edward } & \text { Australia } \\ \text { Boriszewicz } & \text { Contreras Laborca, Carlos (?) } & \text { Chile } \\ \text { Borques, see Borches } & \text { Orechwo, Nikolaj } & \text { Poland } \\ \text { Bronkowski } & & \\ \text { Bueno } & \text { Bortnowski, Bronistaw } & \text { Poland } \\ \text { Chajan, see Chayen } & \text { Calderio, Francisco } & \text { Cuba } \\ \text { Chayen } & & \\ \text { Dolores } & \text { Lé Hông Phong } & \text { Indochina } \\ \text { Draganov } & \text { Ibarruri, Dolores } & \text { Spain } \\ \text { Ercoli } & \text { Stefanov, Boris } & \text { Rumania } \\ \text { Evaristo } & \text { Togliatti, Palmiro } & \text { Italy } \\ \text { Franz } & \text { Medrano, Trifon } & \text { Spain } \\ \text { Funk } & \text { Dahlem, Franz } & \text { Germany } \\ \text { Furini } & \text { Wehner, Herbert } & \text { Germany } \\ \text { Gärtner } & \text { Dozza, Giuseppe } & \text { Italy } \\ \text { Garcio } & \text { Schmidt, Elli } & \text { Germany } \\ \text { Garlandi } & \text { Díaz, José } & \text { Spain } \\ & \text { Grieco, Ruggero } & \text { Italy }\end{array}$

10 "Der Siebente Weltkongress der Kommunistischen Internationale", in: Rundschau über Politik, Wirtschaft und Arbeiterbewegung (Basel), Vol. 4 (1935), Nos 34-45, 47, $49-50,52,54,56,58,60,62,65-66,72,74$. 
Gorkić

Hadjar

Hadschar, see Hadjar

Henderson

Henrykowski

Janaka, see Tanaka

Jussuf

Kansin, see Kon Sin

Karl

Keyros, see Queiros

Kon Sin

Krumov

Kuus

Leński

Martyn

Michal

Nadir

Nisikawa

Okano

Ordeleanu

Pjasecki

Queiros

Raymond

Rwal

Schmidt

Siroký, ${ }^{11}$ see Syrový

Stein

Syrový

Tambay

Tambe, see Tambay

Tanaka

\section{Tanaka}

Tschou Ho Sin, see Tu Ho-shin

\section{Tu Ho-sin}

Ventura

Walter

Wang Ming

Wang Tan

Weber

Wieden
Cižinski, Josip

Halik Wilad, Mahmud

Mpama, Josie (?)

Amsterdam, Saul

Ridwan al Hilu

Hähnel, Walter

Kang Sheng

Sitev, Ilja

Sakkart, Albert

Leszczyński, Julian

Ozol, Martinš

Löwy-Wolf, Hermann Michal

Bagdaš, Chalid

Kobayashi Yonosuke

Nosaka Sanzo

Goldberger, Nicolai

Zander, Nathan

Gonçalves, Bento Antonio

Guyot, Raymond

Reicher, Gustaw

Parović, Blagoje

Siegmund, Kurt

Borkanjuk, Olexo

Bradley, Benjamin F.

Yamamoto Kenzo

No Kao Tsi-li

Hernandes, Jesús

Ulbricht, Walter

Chen Shao-yü

Ha-Huy-Ta

Wiatrek, Heinrich

Fischer, Ernst
Yugoslavia

Palestine

S.Africa

Poland

Palestine

Germany

China

Bulgaria

Estonia

Poland

Latvia

Czechoslovakia

Syria

Japan

Japan

Rumania

Poland

Portugal

France

Poland

Yugoslavia

Germany

Czechoslovakia

India

Japan

China

Spain

Germany

China

Indochina

Germany

Austria

11 Olexa Borkanjuk, who acted at the Congress under the pseudonym Syrový, is mistakenly called Siroký in the source, No 54, p. 2265. The person in question is not Viliam Siroký, who attended the same Congress under his own name, see ibid., No 72, p. 2794. 
1st enlarged plenum (24 February - 4 March 1922) ${ }^{12}$

$\begin{array}{lll}\text { Antonowicz } & \text { Brun, Julian } & \text { Poland } \\ \text { Borodin } & \text { Grusenberg, Michail M. } & \text { Russia } \\ \text { Carr } & \text { Katterfeld, Ludwig } & \text { USA } \\ \text { Jansen } & \text { Proost, Johannes } & \text { Holland } \\ \text { Marshall } & \text { Bedacht, Max } & \text { USA } \\ \text { Stanić } & \text { Novaković, Kosta } & \text { Yugoslavia } \\ \text { Walecki } & \text { Horwitz, Maksymilian } & \text { Poland }\end{array}$

2nd enlarged plenum and proceedings of the ECCI Presidium (6 March - 11 June 1922$)^{13}$

$\begin{array}{lll}\begin{array}{l}\text { Avigdor } \\ \text { Carlo }\end{array} & \text { Kossoi, Jechiel } & \text { Egypt } \\ \text { Cook } & \text { Ljubarskij, Nikolaj M. } & \text { Russia } \\ \text { Jansen } & \text { Cannon, James P. } & \text { USA } \\ \text { Moore } & \text { Proost, Johannes } & \text { Holland } \\ \text { Nicolini } & \text { Ballam, John J. } & \text { USA } \\ \text { P. } & \text { Ljubarskij, Nikolaj M. } & \text { Russia } \\ \text { R. } & \text { Pogány, József } & \text { Hungary } \\ \text { Walecki } & \text { Rudas, László } & \text { Hungary } \\ \text { Warski } & \text { Horwitz, Maksymilian } & \text { Poland } \\ \text { Ziegler } & \text { Warszawski, Adolf } & \text { Poland } \\ & \text { Kurella, Alfred } & \text { Germany }\end{array}$

3rd enlarged plenum (12-23 June 1923)

Aoki

Beruzzi

Brand

Giacomo

Jansen

Krajewski

Laursen

Negri

Saitta
Arahata Katsuzo

Manuil'skij, Dimitrij Z.

Lauer, Henryk

Rákosi, Mátyás

Proost, Johannes

Stein, Wladyslaw

Larsen, Axel

Scoccimarro, Mauro

Vota, Antonio
Japan

UkrSSR

Poland

Hungary

Holland

Poland

Denmark

Italy

Italy

12 Die Taktik der Kommunistischen Internationale gegen die Offensive des Kapitals. Bericht über die Konferenz der Erweiterten Exekutive der Kommunistischen Internationale, Moskau, vom 24. Februar bis 4. März 1922 (Hamburg, 1922).

13 Bericht über die Tätigkeit des Präsidiums und der Exekutive der Kommunistischen Internationale für die Zeit vom 6. März bis 11. Juni 1922 (Hamburg, 1922).

14 Protokoll der Konferenz der Erweiterten Exekutive der Kommunistischen Internationale, Moskau, 12.-23. Juni 1923 (Hamburg, 1923). 
Serra

Stirner

Sultan-Zade

Urbani

Vladetić
Tasca, Angelo

Woog, Edward

Mikaeljan, Avetis

Terracini, Umberto

Cvijić, Djuro
Italy

S.America

Persia

Italy

Yugoslavia

4th enlarged plenum (12-13 July 1924)

Bošković

Ercoli

Freimuth

Laursẹn

Rienzi

Rossi
Filipović, Filip

Togliatti, Palmiro

Remmele, Hermann

Larsen, Axel

Tasca, Angelo

Grieco, Ruggero
Yugoslavia

Italy

Germany

Denmark

Italy

Italy

5th enlarged plenum (21 March - 6 April 1925) ${ }^{16}$

$\begin{array}{lll}\text { Bošković } & \text { Filipović, Filip } & \text { Yugoslavia } \\ \text { Broniewicz } & \text { Sakowski, Leon } & \text { Poland } \\ \text { Domski } & \text { Stein, Henryk } & \text { Poland } \\ \text { Dorsey } & \text { Foster, William Z. } & \text { USA } \\ \text { Hadić } & \text { Mitić, Ljubomir } & \text { Yugoslavia } \\ \text { Hanjes } & \text { Ibanez, Jesús } & \text { Spain } \\ \text { Jack } & \text { Fiedler, Franciszek } & \text { Poland } \\ \text { Krajewski } & \text { Stein, Wladysław } & \text { Poland } \\ \text { Marek } & \text { Dimitrov, Stanke } & \text { Bulgaria } \\ \text { Markowski } & \text { Lampe, Alfred } & \text { Poland } \\ \text { Mehring } & \text { Mirring, Richard } & \text { Estonia } \\ \text { Pepper } & \text { Pogány, József } & \text { USA } \\ \text { Petrov } & \text { Raskol'nikov, Fedor F. } & \text { USSR } \\ \text { Petrovskij } & \text { Pojel, Karl } & \text { Lithuania } \\ \text { Powers } & \text { Lovestone, Jay } & \text { USA } \\ \text { Reinhardt } & \text { Abusch, Alexander } & \text { Germany } \\ \text { Sanborn } & \text { Ruthenberg, Charles E. } & \text { USA } \\ \text { Simić } & \text { Marković, Sima } & \text { Yugoslavia } \\ \text { Tellini } & \text { Oberti, Antonio } & \text { Italy } \\ \text { Ursu } & \text { Antip-Tkačenko, Pavel } & \text { Rumania } \\ 15 \text { Protokoll Fünfter Kongress, op. cit., II, pp. 1028-44. } & \\ 16 \text { Protokoll der Erweiterten Exekutive der Kommunistischen Internationale, Moskau, } \\ \text { 21. März-6. April 1925 (Hamburg, 1925); Exécutif élargi de l'Internationale Commu- } \\ \text { niste. Compte rendu analytique de la session du 21 mars au 6 avril 1925 (Paris, 1925). In } \\ \text { the French version the pseudonym Misković is used for Miljuković (Yugoslavia) and the } \\ \text { pseudonym Morelli for Mauro Scoccimarro (Italy). } & \end{array}$


Viola

Vladetić

Wilkowski, see Witkowski

Witkowski

Landy, Adam

Flecchia, Vittorio

Cvijić, Djuro

Italy

Yugoslavia

Poland

6th enlarged plenum (17 February - 15 March 1926) ${ }^{17}$

Braco

Braun

Domski

Dorsey

Ercoli

Hayashi

Ognjanović

Pepper

Redens

Sanborn

Stefański
Grieco, Ruggero

Ewert, Arthur

Stein, Henryk

Foster, William Z.

Togliatti, Palmiro

Tokuda Kyuichi

Jovanović, Rojko

Pogány, József

Bernstein, Mieczyslaw

Ruthenberg, Charles E.

Danieluk, Aleksander
Italy

Germany

Poland

USA

Italy

Japan

Yugoslavia

USA

Poland

USA

Poland

7 th enlarged plenum (22 November - 16 December 1926) ${ }^{18}$

$\begin{array}{lll}\text { Badulescu } & \text { Moskovici, Gelbert } & \text { Rumania } \\ \text { Baltazar } & \text { Köblös, Elek } & \text { Rumania } \\ \text { Birch } & \text { Lovestone, Jay } & \text { USA } \\ \text { Bošković } & \text { Filipović, Filip } & \text { Yugoslavia } \\ \text { Brand } & \text { Lauer, Henryk } & \text { Poland } \\ \text { Cavalla } & \text { Reggiani, Carlo } & \text { Italy } \\ \text { Duncan } & \text { Minor, Robert } & \text { USA } \\ \text { Ercoli } & \text { Togliatti, Palmiro } & \text { Italy } \\ \text { Ichtiar } & \text { Awerbach, Wolf } & \text { Palestine } \\ \text { Leński } & \text { Leszczyński, Julian } & \text { Poland } \\ \text { Maggi } & \text { Gennari, Egidio } & \text { Italy } \\ \text { Maksymowicz } & \text { Sawrycz, Karol } & \text { Poland } \\ \text { Nikolaević } & \text { Horvatin, Kamilo } & \text { Yugoslavia } \\ \text { Omura } & \text { Takahashi Sadaki } & \text { Japan } \\ \text { Pepper } & \text { Pogány, Jósef } & \text { USA } \\ \text { Petrov } & \text { Raskol'nikov, Fedor F. } & \text { USSR } \\ \text { Turjanski } & \text { Kuźma, Jaroma } & \text { Poland } \\ \text { Vasilkiv } & \text { Kriłyk, Osip } & \text { Poland } \\ \text { 17 Protokoll Erweiterte Exekutive der Kommunistischen Internationale, Moskau, 17. } \\ \text { Febr. bis 15. März 1926 (Hamburg, Berlin, 1926). } \\ \text { 18 Protokoll Erweiterte Exekutive der Kommunistischen Internationale, Moskau, 22. } \\ \text { November - 16. Dezember 1926(Hamburg, Berlin, 1927). }\end{array}$


8th plenum (18-30 May 1927) 19

$\begin{array}{lll}\text { Braun } & \text { Ewert, Arthur } & \text { Germany } \\ \text { Ercoli } & \text { Togliatti, Palmiro } & \text { Italy } \\ \text { Pepper } & \text { Pogány, József } & \text { USA } \\ \text { Petrov } & \text { Raskol'nikov, Fedor F. } & \text { USSR }\end{array}$

9th plenum (9-25 February 1928) ${ }^{20}$

\begin{tabular}{|c|c|c|}
\hline Bennett & Lipec, David & Britain \\
\hline Braun & Ewert, Arthur & Germany \\
\hline \multicolumn{3}{|c|}{ Darsey, see Dorsey } \\
\hline Dorsey & Foster, William Z. & USA \\
\hline Ercoli & Togliatti, Palmiro & Italy \\
\hline Maggi & Gennari, Egidio & Italy \\
\hline Pepper & Pogảny, József & USA \\
\hline
\end{tabular}

10th plenum (3-19 July 1929) 21

\begin{tabular}{|c|c|c|}
\hline Banderas & Pestkowski, Stanisław & Poland \\
\hline Bennett & Lipec, David & USSR \\
\hline Bošković & Filipović, Filip & Yugoslavia \\
\hline Chang Piao & Chang Kuo-tau & China \\
\hline \multicolumn{3}{|c|}{ Dsjui Wito, see Tsju Vito } \\
\hline Ercoli & Togliatti, Palmiro & Italy \\
\hline Garlandi & Grieco, Ruggero & Italy \\
\hline Gorkić & Cižinski, Josip & Yugoslavia \\
\hline Ledo-Amerigo & Lacerda, Fernando & Brazil \\
\hline Leo & Häbich, Walter & Germany \\
\hline Lovera & Amadesi, Luigi & Italy \\
\hline Mif & Firman, Michail & USSR \\
\hline Tanaka & Yamamoto Kenzo & Japan \\
\hline \multicolumn{3}{|c|}{ Tschangpiau, see Chang Piao } \\
\hline Tsju Vito & Chü Chiu-pai & China \\
\hline Witkowski & Landy, Adam & Poland \\
\hline
\end{tabular}

${ }^{19}$ Die Chinesische Frage auf dem 8. Plenum der Exekutive der Kommunistischen Internationale, Mai 1927 (Hamburg, Berlin, 1928).

20 Communist policy in Great Britain. The Report of the British Commission of the Ninth Plenum of the Comintern (London, 1928).

${ }^{21}$ Protokoll 10. Plenum des Exekutivkomitees der Kommunistischen Internationale, Moskau, 3. Juli 1929 bis 19. Juli 1929 (Hamburg, Berlin, 1929). 
11th plenum (25 March - 13 April 1931) 22

Bogen
Bojko
Bošković
Bratkowski
Chuan Pin
Ercoli
Farkas
Garlandi
Henrykowski
Horenko
Kowalczuk
Leński
Mehring
Narayan
Ryng
Sultan-Zade
Walecki

Dua, Gerszon

Vasilev, Ilja

Filipović, Filip

Sochacki, Jerzy

Huang Ping

Togliatti, Palmiro

Poll, Sándor

Grieco, Ruggero

Amsterdam, Saul

Mazur, Franciszek

Hiler, Aleksander

Leszczyński, Julian

Mirring, Richard

Tagore, Saumendra Nath

Heryng, Jerzy

Mikaeljan, Avetis

Horwitz, Maksymilian
Poland

Bulgaria

Yugoslavia

Poland

China

Italy

Hungary

Italy

Poland

Poland

Poland

Poland

Estonia

India

Poland

Persia

Poland

\section{2th plenum (27 August - 15 September 1932) $)^{23}$}

Aki

Antonio

Bratkowski

Bronkowski

Ercoli

Furini

Gallo

Henrykowski

Leński

Maria

Okano

Pringle

Randolph

Svábová
Yamamoto Masami

Gonçalves, Bento Antonio

Sochacki, Jerzy

Bortnowski, Bronislaw

Togliatti, Palmiro

Dozza, Giuseppe

Longo, Luigi

Amsterdam, Saul

Leszczyński, Julian

Filipovici, Elena

Nosaka Sanzo

Gebert, Bolesław

Weinstone, William ${ }^{24}$

Svermová, Marie
Japan

Portugal

Poland

Poland

Italy

Italy

Italy

Poland

Poland

Rumania

Japan

USA

USA

Czechoslovakia

22 XI Plenum IKKI, Stenograficeskij otčet (Moscow, 1931-32).

23 XII Plenum IKKI, Stenografičeskij oťcet (Moscow, 1933).

24 In his article "35 lat w USA", in: Z Pola Walki, II (1959), No 4, p. 142. Boleslaw Gebert states that at the 12th ECCI plenum Robert Minor acted under the pseudonym Randolph. From the minutes of the sixteenth congress of the CPSU it is clear, however, that Randolph was the pseudonym of William W. Weinstone. Sestnadcatyj s"ezd VKP(b), Stenografičeskij otcet (Moscow, 1931), p. 777. 
Wang Ming

Wiktorowicz
Chen Shao-yü

Zambrowski, Roman
China

Poland

13th plenum (28 November - 12 December 1933$)^{25}$

$\begin{array}{lll}\text { André } & \text { Cvijić, Stjepan } & \text { Yugoslavia } \\ \text { Dybov } & \text { Dybov-Planinski } & \text { Bulgaria } \\ \text { Ercoli } & \text { Togliatti, Palmiro } & \text { Italy } \\ \text { Grigorescu } & \text { Danieluk, Aleksander } & \text { Rumania } \\ \text { Jaroslav } & \text { Schram, Augustin } & \text { Czechoslovakia } \\ \text { Kan Sin } & \text { Kang Sheng } & \text { China } \\ \text { Krajewski } & \text { Stein, Wladysław } & \text { Poland } \\ \text { Krause } & \text { Deglavs, Fricis } & \text { Latvia } \\ \text { Leński } & \text { Leszczyński, Julian } & \text { Poland } \\ \text { Michal } & \text { Wolf, Michal } & \text { Czechoslovakia } \\ \text { Mif } & \text { Firman, Michail } & \text { USSR } \\ \text { Okano } & \text { Nosaka Sanzo } & \text { Japan } \\ \text { Oldner } & \text { Goldhammer, Géza } & \text { Czechoslovakia } \\ \text { Rajić } & \text { Čžinski, Josip } & \text { Yugoslavia } \\ \text { Richter } & \text { Schubert, Hermann } & \text { Germany } \\ \text { Roncoli } & \text { Montagnana, Mario } & \text { Italy } \\ \text { Szekerski } & \text { Szpiegel, Meier } & \text { Poland } \\ \text { Walecki } & \text { Horwitz, Maksymilian } & \text { Poland } \\ \text { Wang Ming } & \text { Chen Shao-yü } & \text { China }\end{array}$

${ }^{25}$ XIII Plenum IKKI, Stenograficeskij otčet (Moscow, 1934). 